\title{
An axiomatic approach to a cork granulation facility design
}

\author{
Diogo Martins ${ }^{1}$, João Fradinho, ${ }^{2,}$, Miguel Cavique ${ }^{3}$, António Gabriel-Santos ${ }^{2}$, Alberto Martinho ${ }^{2}$ and António Mourão ${ }^{2}$ \\ ${ }^{1}$ DEMI, Faculdade de Ciências e Tecnologia, Universidade Nova de Lisboa, Campus de Caparica, 2829-516 Caparica, Portugal \\ ${ }^{2}$ UNIDEMI \& DEMI, Faculdade de Ciências e Tecnologia, Universidade Nova de Lisboa, Campus de Caparica, 2829-516 Caparica, \\ Portugal \\ ${ }^{3}$ UNIDEMI, CINAV \& Escola Naval, Base Naval de Lisboa - Alfeite, 2810-001 Almada, Portugal
}

\begin{abstract}
In the conceptual step of any industrial design, there is a considerable amount of decisions that directly affect the quality of the final product. These decisions are often made with lack of knowledge about the subjects, limitative trains of thought and repetition of traditional solutions. The present paper is focused on the use of Axiomatic Design in the design of a cork granulation industrial plant, with a particular emphasis on the Independence Axiom. An innovative design solution was made, aiming to reduce or overcome the current issues and to reevaluate many of the decisions made in the past. In this study, some parts of the production process were analysed. The verified lack of independency gave rise to new design solutions and consequently to a better production process.
\end{abstract}

\section{Introduction}

From the design of machines [1], the optimization of medical surgery sessions [2], or the management of healthcare systems [3], many are the areas in which Axiomatic Design (AD) has proven its usefulness. This paper intends to be another good example of its versatility by exploring the application of $\mathrm{AD}$ in an engineering design, particularly in the design of a cork granulation industrial plant.

The present work starts with a small introduction to the cork granulation process in its current state, going through the used raw materials, its basic principles and a simplistic glance at the existing transformation operations.

Some specific parts of the whole process are explained on a basis of AD. Each case will be analyzed as it is currently done in the traditional cork industry and described through a design matrix. The main issues and faults in these systems will be stated, followed by an alternative design to respond to such problems. The new designs will then be shown in an equation regarding the changes in both functional requirements (FRs) and design parameters (DPs) [4].

\section{The cork granulate process}

The cork granulate process can be viewed as a relatively simple process. There are some differences between competing companies, but overall there are very few variations between each industrial plant.

The cork used to feed the process can have various origins, varying in quality and shape [5]. The three main sources of cork for granulation are cork planks, which is a type of cork that doesn't have the required thickness or quality to be used in the manufacturing of cork stoppers, cork trims, originated from the production of natural cork stoppers, and disc scraps, which are the leftovers from the production of cork discs.

These materials are usually fed into a buffer that will continuously supply the first mill. This machine will break the input material into an aggregate of smaller pieces of cork, which is called "Broken". The maximum dimension of these grains varies from 30 to 50 millimeters, depending on the manufacturer. The dust that's produced at this stage is removed from the process through pneumatic aspiration.

Instead of proceeding straight to the next milling phase, the "Broken" goes through a screening machine, generally, a vibrating screener, which will separate the granulates in two dimensions.

The cork is then guided into different milling machines, which are adjusted to their optimal working condition according to grain size, and thus creating less dust and improving efficiency. This second phase of the reduction of the cork size transforms the cork into its final granulate size. Much tighter screens are used, producing grain sizes ranging from 0.5 to 7 millimeters [6].

The number of used screening machines and mills, as well as grain size range, may vary amongst manufacturers. Some plants use parallel production lines to improve their capability.

Once the granulates satisfy the dimensions imposed by the mill screens, the cork proceeds to a drum dryer to achieve the right humidity content. Humidity is a very

\footnotetext{
* Corresponding author: jimf@,fct.unl.pt
} 
important topic as the right water content will determine the quality of the products that the granulates will produce. The drying operation can be executed in a single stage or along the process in multiple dryers. Some facilities dry their cork as "Broken" instead of in its final form.

Being a natural product that needs 9 years to be harvested, all sources of cork have a certain impurity content which is practically impossible to remove. Cleaner and lighter cork has more market value than lower quality and unclean cork. For that reason, after being dried to an approximate humidity content of $14 \%$, there is a need to separate the cork granules according to their specific weight. To do so, the bulk of granulates goes through another set of screening machines and is divided into small granulometry intervals. The common intervals to be found are: $[0.5 ; 1],[1 ; 2],[2 ; 3],[3 ; 5]$ and $[5 ; 7]$ in millimeters. At this stage, grains smaller than 0.5 millimeters are considered dust and are removed from the process. Grains larger than 7 millimeters are reintroduced in the previous mills.

The density separation itself is made through densimetric tables. By using streams of air and vibration, alongside with a small inclination of the table plate, these machines separate heavier and dirt granules from the clean and lighter ones. Ideally, the output of this process will be granules rounding specific weights of about 50 to $70 \mathrm{~kg} / \mathrm{m} 3$ as the best quality, and from 70 to $100 \mathrm{~kg} / \mathrm{m} 3$ as medium quality products. Above those sizes, the granules are reintroduced in the previous mills, as to extract the maximum amount of clean cork possible.

The processed granulates are then guided to silos or stored in Big Bags.

In most facilities, the transportation of cork between the different stages is made through pneumatic or gravity conveyors. These plants are typically built in height rather than in length.

The dust produced along the process is collected into silos and can be sold as a product or used in furnaces to generate thermal energy for the plant.

A flowchart of the process can be seen in Figure 1.

\section{Improvements made through the use of Axiomatic Design}

Being a process that has had few innovations in the past decades, few modern and innovative ideas were considered in the existing industrial layouts. In fact, many of these plants grew thanks to small improvements to the original ones. This lead to many coupled systems and therefore many opportunities for improvement.

Axiomatic Design is the tool that was used in this paper to explore some of the main issues that have been found in the lifetime of these processes. Using the AD's concept of independence, better designs were attained. In this section, the current condition, as well as the several issues of the cork granulation industries are analyzed. and new proposals are presented.

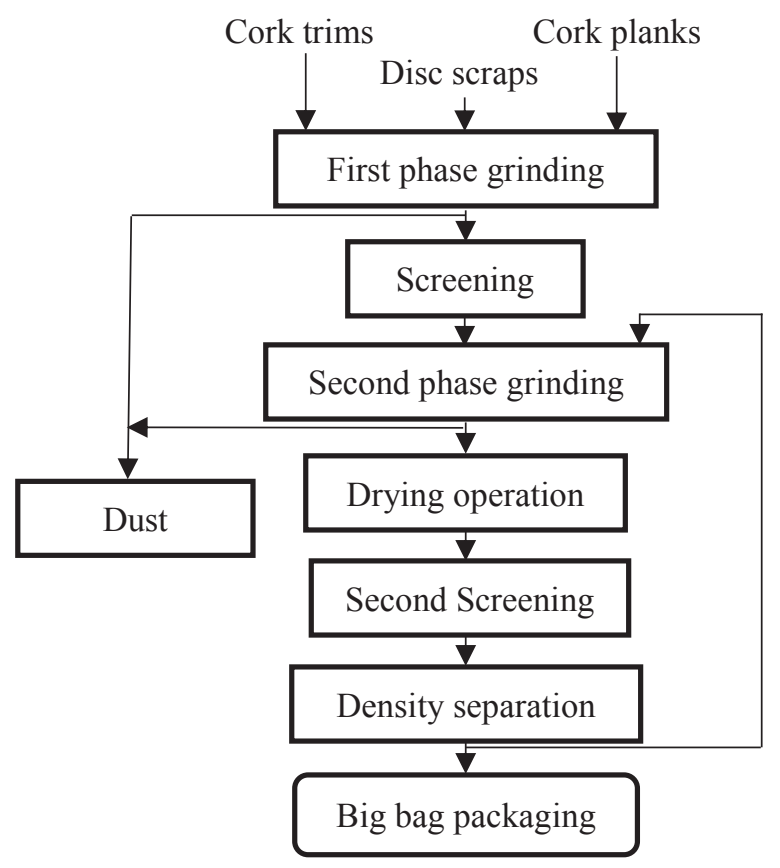

Fig. 1. Flowchart of the cork granulation process

This study is based on the relations between the functional and the physical domains. The functional requirements derive from the customer needs (CNs), which in this case is the owner of the industrial facility, who wants to achieve a good yield in the production of cork granules within certain dimension and density ranges, as to be used mainly in his cork stoppers plants. It is worth noting that the relations between CNs and FRs are "loosely structured", as noted by Harutunian et al. [7].

\subsection{Raw material storage}

Starting from the very beginning, there's a critical issue in the traditional cork granulating processes related to the control of the fraction of the three different raw materials that are referred in the previous section. The source of this problem can be traced to the shared storage area used for different raw materials.

The mixing of the various types of cork will result in an unstable proportion in the cork feed over time. This will lead to a fluctuation in the final blend that will affect in a negative way the efficiency of the process machinery and, thus, of the whole plant.

In the viewpoint of $\mathrm{AD}$, it's easy to see beforehand that this problem will result in coupled designs in the following stages of the process.

Regarding this issue, and through the analysis of the cork feed operation, the following functional requirements are defined:

$F R_{1}$ - Ensure the continuous feed of cork

$F R_{2}$ - Minimize transportation times

$F R_{3}$ - Minimize the variability of cork types in the process

The answers to these requirements are currently given by the following design parameters: 
$D P_{1}$ - Wheel loading shovel

$D P_{2}$ - Distance between the piles of cork and the first mill

The following design equation is representative of the process in hand:

$$
\left\{\begin{array}{l}
F R_{1} \\
F R_{2} \\
F R_{3}
\end{array}\right\}=\left\{\begin{array}{ll}
\times & 0 \\
0 & \times \\
\times & 0
\end{array}\right\}\left\{\begin{array}{l}
D P_{1} \\
D P_{2}
\end{array}\right\}
$$

AD's Theorem 1 states, "when the number of DPs is less than the number of FRs, either a coupled design results or the FRs cannot be satisfied" [8].

The current case represents a coupled design and consequently does not correspond to a good design.

A possible alternative to this system is to separate the different types of cork into distinct piles at arrival, using either different storage locations or using separate compartments in the same storage unit.

Due to the average production rate of a cork granulating process, storing all the cork in the same location would require a huge warehouse. On the other hand, having the cork scattered in different locations would jeopardize the fulfillment of $F R_{2}$.

In this scenario, the best solution is to aggregate both alternatives stated above, i.e. store the various types of cork in separate locations, and create an intermediate buffer with compartments for each type of cork near the first mill.

These changes will create an additional design parameter:

$D P_{3}$ - Separation of input materials storage by type

The result is the following design equation:

$$
\left\{\begin{array}{l}
F R_{1} \\
F R_{2} \\
F R_{3}
\end{array}\right\}=\left\{\begin{array}{ccc}
\times & 0 & 0 \\
0 & \times & 0 \\
\times & 0 & \times
\end{array}\right\}\left\{\begin{array}{l}
D P_{1} \\
D P_{2} \\
D P_{3}
\end{array}\right\}
$$

This simple modification of the plant, allow achieving a decoupled design and therefore a better overall design.

\subsection{Cork fraction control}

Although the supply of the different types of cork was designed in the last section to be made independent through the addition of an extra design parameter, there is still an issue with controlling the fractions of the final cork blending used in the process.

As the cork is supplied to the mill by using a loader, the quantities that are fed each time the operator drops a cargo differ from the previous ones. Since the loader is hand-operated, then the fractions of the different types of cork will vary according to the operator's skills and experience.

Analyzing the feeding sequence through the $\mathrm{AD}$ viewpoint, the following functional requirements can be attained:
$F R_{1}$ - Ensure the required feed of cork into the process

$F R_{2}$ - Process the cork into smaller pieces

$F R_{3}$ - Keep steady the fractions of the different cork types over time.

$F R_{4}$ - Adjust the fractions of the three types of cork

The answers to these requirements in the traditional facilities are given by the following design parameters:

$$
\begin{aligned}
& D P_{1} \text { - Full-time loader operator } \\
& D P_{2} \text { - Mill }
\end{aligned}
$$

therefore, following design equation holds.

$$
\left\{\begin{array}{l}
F R_{1} \\
F R_{2} \\
F R_{3} \\
F R_{4}
\end{array}\right\}=\left[\begin{array}{cc}
\times & 0 \\
0 & \times \\
\times & 0 \\
\times & 0
\end{array}\right]\left\{\begin{array}{l}
D P_{1} \\
D P_{2}
\end{array}\right\}
$$

Eq. (3) portrays a coupled design, as per AD's Theorem 1.

To control the fractions of cork types, it is necessary to completely rearrange the initial layout of the plant.

Cork planks are cheap but unclean, while trims and discs are high quality materials.

Two independent mills would be used to allow the independent milling of the two quality groups of cork, instead of the traditional milling of a multi typed cork blend. This change would significantly improve the control over the final mixture, as it creates two independent flows of material $\left(\mathrm{DP}_{3}{ }^{\prime}\right)$.

Associated with each milling station, a mechanical conveying system would be installed. These two conveyors would converge the two cork flows into the dryer (the next phase of the process) in the required fractions.

Using a variable speed drive $\left(\mathrm{DP}_{4}{ }^{\prime}\right)$ in each conveyor would allow adjusting the feed rate of each cork quality type and, thus, fulfilling $F R_{4}$.

Regarding these new components, a new functional requirement arises at this level:

$F R_{5}$ - Ensure the continuous material feed to the conveyor

The use of silos located after the mills and above the conveyors $\left(D^{2}{ }^{\prime}\right)$, working as buffers, would ensure the continuous feed of the conveyors, fulfilling $F R_{5}$.

Therefore, the new design parameters are defined as follows:

$D P_{1}^{\prime}$ - Full-time loader operator

$D P_{2}^{\prime}$ - Two mills

$D P_{3}^{\prime}$ - Two separate and independent circuits (mill, silo and conveyor)

$D P_{4}^{\prime}$ - Variable speed drives

$D P_{5}^{\prime}$ - Silos over the conveyors

This results in the following design equation: 


$$
\left\{\begin{array}{l}
F R_{1} \\
F R_{2} \\
F R_{5} \\
F R_{3} \\
F R_{4}
\end{array}\right\}=\left[\begin{array}{ccccc}
\times & 0 & 0 & 0 & 0 \\
0 & \times & 0 & 0 & 0 \\
\times & \times & \times & 0 & 0 \\
0 & 0 & \times & \times & 0 \\
0 & 0 & 0 & \times & \times
\end{array}\right]\left\{\begin{array}{c}
D P_{1}^{\prime} \\
D P_{2}^{\prime} \\
D P_{5}^{\prime} \\
D P_{3}^{\prime} \\
D P_{4}^{\prime}
\end{array}\right\}
$$

As shown by Eq. (4), a decoupled design was attained. This represents a better design when compared to the previous coupled design. Nevertheless, some dependencies are important to notice.

First, as to ensure that the mechanical conveying system will actively maintain the fractions of the two cork quality types, a certain amount of cork should be always kept in both buffers. Although each buffer will allow the system to work for a limited period, this will happen only if the associated mill is working and the loader operator is performing accordingly to the plant's needs.

Second, the adjustments on the fractions of the cork types are only assured by the variable speed drive if the mechanical conveyors are operational.

Even though these inconvenient situations could occur, then there is a considerable improvement in the satisfaction of the various functional requirements.

Furthermore, the dependencies that can be found in the design matrix in Eq. (4) are relatively simple to manage.

Another interesting advantage of this system is that it would allow running with different fractions of the various cork types with the final goal of finding an optimal price/quality ratio.

The main causes of stopping the production are maintenance operations and failures of the first grinding stage. This leads to maintenance interventions that can last from a couple of hours to days at a time.

Since the design changes introduced the use of two separate mills, a very suitable approach to minimize the down times of the plant can be implemented.

It would be possible for the plant to work at full speed with one single mill for a limited period through the installation of a diverter connecting each mill to both the silos associated with the fraction control. This period would be mainly defined by the mill maximum speed and the volume of cork that can be stored in each silo.

This way, considering the possibility of processing both quality types of cork in both mills, the fraction control method used before would be assured, even during this temporary production method.

In addition, if the malfunction or intervention requires a much longer period to resolve, one could assure the full speed of the facility by using the same mill for the three types of raw material.

\subsection{Drying efficiency}

One of the most critical characteristics of cork is its humidity content.

If the cork goes into the mills with a high level of moisture, then the cutting tools will suffer more abrasion than in normal operation and will wear out in a much shorter period. On the other hand, if the humidity level is too low, then the separation of the cork grains will release a large amount of cork dust, thus reducing the quantity of grains that are produced. Regarding the final product, the right amount of moisture is crucial in many processes that use cork granulates.

The cork moisture is often higher than the required, so most industrial cork processes rely on drum dryers to lower its value.

Drum dryers use a combination of heat and airflow, as well as a tumbler movement that circulates the cork granulates around the drum's carcass. Heat, airflow and movement are responsible for removing water from the cork, as well as for the power consumption of the drum dryer. Having the dryer set to the minimum temperature and airflow required to reach the right level of humidity is a big step for having an energy efficient plant.

In the current industry, the humidity control is made through the measurements of samples that are regularly withdrawn from the production line. This slow process results in a considerable delay in the required adjustments of the dryer's operating parameters.

Looking for an alternative approach, the following functional requirements were defined:

$F R_{1}$ - Dry the granules

$F R_{2}$ - Inspect granule moisture

Considering the standard plant design, the following design parameters were considered:

$$
\begin{aligned}
& D P_{1} \text { - Drum dryer } \\
& D P_{2} \text { - Airflow control } \\
& D P_{3} \text { - Temperature control } \\
& D P_{4} \text { - Quality control operator }
\end{aligned}
$$

resulting in the following design equation:

$$
\left\{\begin{array}{l}
F R_{1} \\
F R_{2}
\end{array}\right\}=\left[\begin{array}{llll}
\times & \times & \times & \times \\
0 & 0 & 0 & \times
\end{array}\right]\left\{\begin{array}{l}
D P_{1} \\
D P_{2} \\
D P_{3} \\
D P_{4}
\end{array}\right\}
$$

This system represents a redundant decoupled design as it has a right-trapezoidal design matrix [9].

The adjustment of the airflow will impact the temperature of the air that goes into the drum dryer. A higher airflow will result in cooler air and a slower airflow will result in a temperature rise inside the dryer.

In what to drying concerns, the current design parameters were questioned as to achieve a better design.

Instead of having an operator to adjust the various settings of the drum dryer, according to some humidity readings, it would be interesting to use an automated system.

Fourier transform near-infrared (FT-NIR) spectroscopy was taken into consideration to automatically measure humidity fluctuations of the granules. This method is used in some specific applications with granulates such as sugar, green tea leaves, and some pharmaceutical products.

Ideally, there would be two humidity measuring devices, one at the entry and the other at the exit of the 
drum dryer. The difference between both levels, as well as the output humidity, would be controlled by a computer program that regulates the temperature and the flow of air that goes into the dryer.

Currently, automated systems to measure the humidity content of cork are not commercially available, but such a method seems to be suitable for this kind of application.

Studies would have to be made to better understand the limitations of such method, if there are any, and to understand its outputs in order to define appropriate parameters' values.

Under these circumstances, the design parameters would be:

\section{$D P_{1}$ - Drum dryer \\ $D P_{2}^{\prime}$-Continuous and automated control of the drying parameters through a system based on FTNIR spectroscopic sensors.}

The resulting design equation is the following:

$$
\left\{\begin{array}{l}
F R_{1} \\
F R_{2}
\end{array}\right\}=\left[\begin{array}{ll}
\times & \times \\
0 & \times
\end{array}\right]\left\{\begin{array}{l}
D P_{1} \\
D P_{2}^{\prime}
\end{array}\right\}
$$

The design matrix of Eq. (6) represents a decoupled design, since its design matrix is triangular.

There is no evidence of any work done to support the usage of the FTNIR spectroscopy in the cork industry.

\subsection{Densimetric table optimization}

Gravity tables are used to separate different density intervals of cork granulates into three different grades: light and clean cork, slightly dirty and medium weighted cork; and, at last, dirty and heavy cork.

The characteristics of each grade will determine the price range to which buyers are willing to pay for. For instance, the dirtier and heavier cork has such a low market value that it is often reintroduced in the mills to try removing any smaller pieces of clean cork that are embedded in the dirty grains. For this reason, the efficient separation of the three grades is an important subject as to ensure the maximum profit.

The current industry often presents fluctuations in each cork grade. This is due to the high sensitivity to small adjustments of the machines. Additionally, it is very difficult to fully understand how the design parameters will affect the efficiency of the cork separation.

Regarding the need for a more efficient process, the following functional requirements were defined:

$F R_{1}$ - Separate the various cork grades

$F R_{2}$ - Adjust working parameters

Considering the standard plant design, the following design parameters were considered:

$D P_{1}$ - Densimetric table

$D P_{2}$ - Control devices

The resulting design equation is the following:

$$
\left\{\begin{array}{l}
F R_{1} \\
F R_{2}
\end{array}\right\}=\left[\begin{array}{ll}
\times & \times \\
0 & \times
\end{array}\right]\left\{\begin{array}{l}
D P_{1} \\
D P_{2}
\end{array}\right\}
$$

At a lower level of decomposition, the parameters that define the efficiency of the densimetric tables are the airflow and the flow rate of the granules, as well as the longitudinal and transverse inclination of the table. In a usual layout, the adjustable parameters (table inclination and air flow) are hand-controlled, at the mercy of human mistake or misusage. Therefore, their impact on the efficiency of the process depends on the operator.

Regarding the cork granulate feed rates, they are imposed by the amount of cork produced in the mills, which is directly conveyed to the tables. The variability of the input materials will lead to the inconsistency of the feed rates, resulting in a recurring excess or lack of material in the machines. Since the working principle of these machines is the sliding of lighter granules over the heavier ones, an unbalanced feed rate will lead to fluctuations in the cork grades previously defined. For this reason, the densimetric tables are very sensitive to the amount of granules that they are separating.

According to the previous statements, even though the current design is decoupled, there is a manner to achieve a better result, by adding two more FRs, as follows:

$F R_{3}$ - Memorize working set-ups

$F R_{4}$ - Control the cork feed rate

Switching the man-operated controls for an electronic system panel makes small adjustments easier. Furthermore, this system would allow creating a database of the various set-ups and standard combinations for each particular cork blend.

Other issues associated with adjusting the parameters in the traditional cork processes are the blend differences over time. The changes in the initial milling operation described in section 3.2 are bringing benefits to this operation as well, improving the overall process.

Another issue to resolve is the unsteady feed of the cork granulates into the tables. With the introduction of a buffer with a flow-restricting valve, a very common system in other industries, there would be a bettercontrolled feed to the tables.

This new ideas and systems will result in the addition of the following design parameters:

$D P_{3}$ - Electronically controlled system

$\mathrm{DP}_{4}$ - Buffer over each table

Thus, the next design equation holds:

$$
\left\{\begin{array}{l}
F R_{1} \\
F R_{2} \\
F R_{3} \\
F R_{4}
\end{array}\right\}=\left[\begin{array}{llll}
\times & \times & 0 & 0 \\
0 & \times & 0 & 0 \\
0 & 0 & \times & 0 \\
0 & 0 & 0 & \times
\end{array}\right]\left\{\begin{array}{l}
D P_{1} \\
D P_{2} \\
D P_{3} \\
D P_{4}
\end{array}\right\}
$$

As it can be seen in the design matrix of Eq. (8), a decoupled design is attained. The addition of two functional requirements with the matching design parameters will improve the separation efficiency. The 
present design represents a better design than the traditional.

\section{Conclusions}

The Axiomatic Design theory is a very powerful tool to achieve good designs for any kind of component or system. This paper presents another good example of its versatility.

In a direct application regarding the very particular industry of cork granulation, a considerable amount of issues were minimized or even resolved through the adaptation of the existing layouts to the axiomatic perspective.

It is visible that using this theory in the initial stages of a big scale industry project such as this one, will greatly improve its overall efficiency as well as many other important factors such as management operations and quality control.

In this work, four sub-processes of the cork grinding were analyzed. All of them showed lack of independency and were redesigned to fix this issue. The previous and the proposed design matrixes were presented.

The main changes that were considered in the various phases of the process are of relatively simple nature, since building the systems from scratch won't represent a major difficulty. On the other hand, changing the existing layouts to fit these new considerations would be costly and required a lot craftmanship and engineering. This is yet another indicator that design theories have a major role in the earlier stages of any design. Furthermore, the axiomatic approach may sometimes have positive impacts in the following stages, as shown in section 3.4.

The difference in the initial investment (machines, equipment and assembly) of the new design in comparison to the traditional vision is considerable, particularly in the cork fraction control system. However, given that the proposed modifications lead to a decoupled solution, then it is always a better solution than the traditional coupled one, as per Axiomatic Design viewpoint.

The authors gratefully thank the sponsorship of Fundação para a Ciência e Tecnologia through the Strategic Project UID/EMS/00667/2013 - UNIDEMI.

\section{References}

1. J. W. Melvin, Axiomatic System Design: Chemical Mechanical Polishing Machine Case Study. PhD Thesis, MIT, Dept. of Mechanical Engineering (2003).

2. D. Bottacci, P. Citti, A. Giorgetti, "Development of a tailored tool for the optimization of the surgery sessions", Proc. 5th Conf. on Axiomatic Design (ICAD 2009), p. 85-91, Campus de Caparica, Portugal (2009).
3. H. L. Oh, "Scheduding pre-admission testing: A case study on reducing complexity in heralthcare delivery", Proc. $6^{\text {th }}$ Conf. on AxiomaticDesign (ICAD 2011), p. 170-176. Daejeon, Rep. of Korea (2011).

4. M. K. Tompson, "A classification of procedural errors in the defenition of functonal requirements in Axiomatic Design Theory", Proc. $7^{\text {th }}$ Conf. on Axiomatic Design (ICAD 2013), Worcester, USA (2013)

5. J. Ferreira, Contributos para o aumento do rendimento de uma unidade de trituração de cortiça, MSc Thesis FCT-UNL (in portuguese) (2017).

6. J. Apolinário, Melhoria da Fiabilidade de uma Unidade de Trituração de Cortiça, MSc Thesis FCTUNL (in portuguese) (2017).

7. V. Haratunian, M. Nordlund, D. Tate, N. P. Suh, "Decision Making and Software Tools for Product Development on Axiomatic Design Theory", CIRP Annals - Manuf. Tech. 45:1, pp. 135-139 (1996).

8. N. P. Suh, The principles of design. Oxford University Press (1990).

9. A. M. Gonçalves-Coelho, G. Neştian, M. Cavique, and A. Mourão, "Tackling with redundant design solutions through Axiomatic Design," Int. J. Precis. Eng. Manuf., 13, 10, pp. 1837-1843 (2012). 